\title{
Analysis of Energy-Efficient, Fair Routing in Wireless Sensor Networks through Non-linear Optimization
}

\author{
Bhaskar Krishnamachari \\ Electrical Engineering-Systems \\ University of Southern California \\ Los Angeles, CA 90089 \\ Email: bkrishna@usc.edu
}

\author{
Fernando Ordóñez \\ Industrial Systems Engineering \\ University of Southern California \\ Los Angeles, CA 90089 \\ Email: fordon@usc.edu
}

\begin{abstract}
In the area of wireless sensor networks (WSN) there is still a significant gap between theory and practice: system designs and protocols are rapidly out-pacing analysis. We develop formal computational models of a WSN based on non-linear optimization and use them to analyze the impact of fairness constraints on network performance. The optimization framework presented is very general and can also be used to analyze the optimal performance of WSN subject to other design parameters such as the topology, number of nodes, energy levels, source rates, reception power, etc. Our results show that the maximum information that can be extracted for a fixed amount of energy increases and that the minimum energy required to output a fixed amount of information decreases as we reduce the fairness requirement in the network. We present these functions for a fixed network topology and observe that they exhibit sharp changes in gradient due to qualitative changes in optimal routes.
\end{abstract}

\section{INTRODUCTION}

Wireless sensor networks (WSN) are an emerging technology which seem ready to revolutionize the availability and quality of information in a wide array of application areas. This new technology has come about due to the rapid advances in embedded microprocessors, wireless communications, and MEMS sensors over the past decade.

As we set out to design and implement these kinds of systems, however, one fact becomes clear. In the area of WSN there is a significant gap between theory and practice: proposed system designs and protocols are rapidly out-pacing mathematical understanding. While there is a strong mathematical foundation for communication over a single link from classical Information Theory, there are very few formal models for analyzing the fundamental performance of information routing in wireless networks.

The broad motivation for this work is this need for meaningful mathematical models of WSN. Such models are necessary to understand the theoretical bounds on performance and the impact of different design parameters such as topology, number of nodes, energy levels and fairness. We take an optimization based modeling approach in this paper. Due to the underlying equations that describe the capacity of physical channels, we will rely on convex non-linear optimization techniques.

It is helpful to place this approach in context by comparing it with the standard approach to design and evaluate protocols for sensor networks. Much of the current literature in sensor and ad-hoc wireless networks consists of practical proposals for new protocols for information routing. Typically simulation results are used to examine the impact of various parameters on the effectiveness of the protocol. Comparisons are usually performed either with some baseline strategies or with alternative protocols. Iterated over time, this procedure yields practical, implementable protocols with successively better performance characteristics. However, if we do not know the fundamental bounds imposed by the underlying problem structure, then it will not be clear how the implemented protocol differs from optimal performance. An estimation of the optimal performance will allow us to determine if there is room for additional improvement of a given protocol ${ }^{1}$. Our approach is to characterize the optimal performance of information routing (under carefully defined assumptions and constraints) without reference to the details of any practical distributed schemes that can realize this performance.

We present some general non-linear optimization models in this paper that can be used to analyze the fundamental limits on the performance of information routing. In particular we explore the fundamental influence of different fairness requirements of the network on the overall performance of the WSN through some simple experiments. We treat end-to-end fairness by placing constraints on the maximum percentage of the total information to the sink that each source node can send. We refer to these constraints as the fairness constraints.

\footnotetext{
${ }^{1} \mathrm{~A}$ good analogy is the classic Channel Coding Theorem; it is only in recent years that iterative decoding mechanisms for Turbo codes and low density parity check codes have been shown to provide near-optimal performance. The bounds developed by Shannon give us confidence that no other (as yet undeveloped) coding/decoding techniques can perform significantly better than these. Of course, physical-layer results from information theory have the additional merit of being closed-form expressions. Given the complexities of the networking wireless nodes, however, we restrict ourselves to non-closedform algorithmic optimization formulations in this work.
} 
Thus, in a completely fair WSN every node contributes the same percentage of the total information to the sink, and in a completely unfair WSN all the information to the sink could be from a single source node.

The rest of the paper is organized as follows. In Section II, we will discuss some recent related work to place our own in context. We then present our general model and optimization formulation in Section III. In Section IV, we present the results of our simple experiments that study the impact of fairness constraints on the energy requirements and total information flow for a few different WSN. Our concluding comments are presented in section $\mathrm{V}$.

\section{RELATED WORK}

Most recent work on sensor networks has been primarily in the form of protocol development, and simulation studies [1]. The body of literature on analysis of the fundamental behavior and limitations of these kinds of wireless networks is considerably smaller.

Related to our approach is the work by Toumpis and Goldsmith on capacity regions for wireless networks [2], [3]. Using a linear-programming optimization based formulation (similar in spirit to our work), the authors study the characteristics of the maximum information throughput that can be obtained in a network with arbitrary topology. Non-linear constraints of the physical channel are taken into account in the work of [4]. One key difference from our work is that these papers focus on general-purpose wireless networks and do not incorporate energy or fairness constraints in their modeling.

Optimization models have also been used to study maximum lifetime conditions for sensor networks. Bhardwaj and Chandrakasan [5] develop upper bounds on the lifetime of networks based on optimum role assignments to sensors (e.g. whether they should act as routers or aggregators). Kalpakis et al. [6] formulate a linear programming problem to schedule flows within the network in such a way as to maximize the network lifetime. Our work is similar to these in that we too employ a flow-based formulation, but we incorporate a number of different constraints such as the non-linear physical channel conditions and also the fairness constraints.

Generally speaking, fairness in resource allocation is an issue that is considered in both end-to-end and medium access (MAC) layer contexts. In the context of sensor networks, Woo and Culler have developed a protocol to provide MAC-level fairness [7]. In this paper we primarily treat end-to-end fairness and place constraints on the maximum percentage of the total information to the sink that each source can send.

\section{Modeling AND Problem Formulation}

In this section we present two optimization based formulations for the wireless sensor network problem. Both models consider $n$ sensor nodes, each with limited energy $E_{i}$ and a maximum source rate of $R_{i}$ (i.e. node $i$ is capable of transmitting at a maximum rate of $R_{i}$ ). The models consider variables $f_{i j}$ and $P_{i j}$ that denote the information flow rate and transmission power on the link between nodes $i$ and $j$ on the network. These variables are used to balance the competing objectives in the WSN of maximizing the amount of information that reaches a sink (node $n+1$ with unlimited energy resources - a reasonable assumption if the sink is "plugged in"), and minimizing the total consumption of energy. The two models differ in how these competing objectives are handled.

Both models also consider $C$ the per-bit rate reception power for each node, $\eta$ the noise in the communication channel, and $d_{i j}$ the physical distance between nodes $i$ and $j$. Additionally the models limit the total amount of data that node $i$ sends to a certain fairness proportion $\alpha_{i}$ of the total information sent to the sink. Finally we assume that all transmissions are scheduled (either through time or frequency division multiplexing) such that they are non-interfering.

\section{A. Formulation (1): Maximizing Information Extraction}

Our first model balances the competing minimum energy and maximum information objectives by limiting the total energy on each sensor node and maximizing the total information that can be extracted. The model below pre-specifies the positions, the energy levels, source rates, and fairness constraints for the nodes and then maximizes the total information routed to the sink.

$$
\begin{array}{ll}
\max & \sum_{j=1}^{n} f_{j n+1} \\
\text { s.t. } & \sum_{j=1}^{n} f_{j i} \leq \sum_{j=1}^{n+1} f_{i j} \\
& \sum_{j=1}^{n} f_{j i}+R_{i} \geq \sum_{j=1}^{n+1} f_{i j} \\
& \sum_{j=1}^{n+1} f_{i j}-\sum_{j=1}^{n} f_{j i} \leq \alpha_{i} \sum_{j=1}^{n} f_{j n+1} \\
& \sum_{j=1}^{n+1} P_{i j}+\sum_{j=1}^{n} f_{j i} C \leq E_{i} \\
& f_{i j} \leq \log \left(1+\frac{P_{i j} d_{i j}^{-2}}{\eta}\right) \\
& f_{i j} \geq 0, P_{i j} \geq 0
\end{array}
$$

In this model, the objective function is the total information that is being routed from all sources to the sink. We now explain each of the constraints in the model. Constraint (1a) ensures that for all nodes except the sink, the out-flow from each node is at least as large as the in-flow. In other words, intermediate nodes do not drop packets ${ }^{2}$. Constraint (1b) is the maximum source rate constraint and says that the outflow from any node is always less than its in-flow plus the maximum rate at which the node obtains information (the source rate $R_{i}$ ).

Constraint (1c) is the fairness constraint, it ensures that each node $i$ cannot generate a flow to the sink that is greater than a fraction $\alpha_{i}$ of the total flow being generated by the network

\footnotetext{
${ }^{2}$ This constraint disallows the possibility of data aggregation, and would consequently have to be modified if we wish to extend our models to allow in-network aggregation
} 


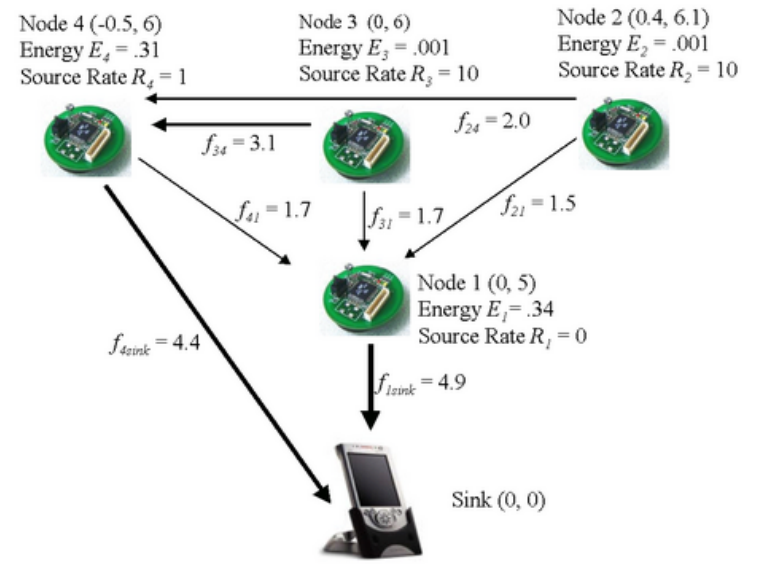

Fig. 1. Illustration of a 5 node wireless sensor network with optimal flows (Not to scale).

to the sink. Note that when $\alpha_{i}=\frac{1}{n}$ for every node $i$, then all nodes send an equal amount of flow to the sink. Constraint (1d) is the energy constraint. We allow each node $i$ to transmit at a different power $P_{i j}$ to other distinct nodes $j$. The total energy expended by each node is a combination of the transmission energy (which depends on the sum of its power settings for transmission to other nodes) and the reception energy which is charged a per-bit cost of $C$. In this model, each node may expend no more than a pre-specified finite amount of energy $E_{i}$.

Constraint (1e) is essentially Shannon's capacity theorem, which relates the maximum flow on a particular channel to the SNR (signal to noise ratio) on that channel. Interference effects are not taken into account in this model, which is reasonable for channelized/scheduled networks - in any case since interference can only make the information flow lower than the optimal, it is reasonable to ignore it to obtain upper bounds. We have modeled the wireless channel as an AWGN channel (which is a reasonable assumption for line-of-sight sensors and low-fading physical environments). The received signal power is the transmit power attenuated by the square of distance between the transmitter and receiver (more generally, we could consider any attenuation exponent that is between 2 and 5 to model the radio propagation conditions). Finally, constraint (1f) ascribes the non-negativity property to the pairwise flow and transmission power variables.

The optimization problem described above is tractable, and current non-linear optimization research software can tackle moderate size versions of this problem. We have obtained preliminary results for it using LOQO, an interior point algorithm that incorporates a merit function to enforce feasibility of the optimal solution [8]. Figure 1 illustrates the solution for a small example. Given the locations of the nodes, and their source rates and energy levels, the flows indicated in the figure maximize the total information that can be collected by the sink before the nodes run out of energy. The optimal solution contains intuitively understandable features - for example, nodes 1 and 4 are selected as intermediate routers for the data from nodes 2 and 3 because they have relatively greater energy resources, and lower source rates.

\section{B. Formulation (2): Minimizing Energy Usage}

The second model balances the competing minimum energy and maximum information objectives by limiting the minimum information to be extracted to the sink and minimizing the energy required to do this. The model below pre-specifies the minimal information $f_{\min }$, and the positions, source rates, and fairness constraints for the nodes and then minimizes the total energy required:

$$
\begin{array}{ll}
\min & \sum_{i=1}^{n}\left(\sum_{j=1}^{n+1} P_{i j}+\sum_{j=1}^{n} f_{j i} C\right) \\
\text { s.t. } & \sum_{j=1}^{n} f_{j i} \leq \sum_{j=1}^{n+1} f_{i j} \\
& \sum_{j=1}^{n} f_{j i}+R_{i} \geq \sum_{j=1}^{n+1} f_{i j} \\
& \sum_{j=1}^{n+1} f_{i j}-\sum_{j=1}^{n} f_{j i} \leq \alpha_{i} \sum_{j=1}^{n} f_{j n+1} \\
& \sum_{j=1}^{n} f_{j n+1} \geq f_{\min } \\
& f_{i j} \leq \log \left(1+\frac{P_{i j} d_{i j}^{-2}}{\eta}\right) \\
& f_{i j} \geq 0, P_{i j} \geq 0
\end{array}
$$

The only differences in this second model are the constraint (2d) on the total information obtained by the sink (which must now be at least $f_{m i n}$ ), and the different objective function: we now minimize total energy usage at all nodes except the sink (which is assumed to have unlimited energy resources). As before there are two components to the energy expenditure at each node: energy expense due to transmission and reception energy. The other constraints (2a), (2b), (2c), (2e), and (2f) are identical to those used in the previous model. Like the first model, current non-linear optimization research software can tackle moderate size versions of this second problem as well.

\section{EXPERIMENTS AND RESUlTS}

We considered a very simple example to explore the fundamental influence that the fairness constraint has on the total information that can be extracted and the amount of energy required and its optimal distribution. Both formulations of the problem, Problems (1) and (2), were used to model a WSN that consisted of a sink and four sensor nodes in line, equally spaced. In these experiments, the four nodes are located at $(1,0),(2,0),(3,0)$ and $(4,0)$ respectively, with the sink at $(0,0)$.

We considered a uniform fairness constraint across all nodes, that is $\alpha=\alpha_{i}$ for all nodes $i$. We gradually increased the fairness constraint between $25 \%$ to $100 \%$ and report its influence on the amount of information extracted and the minimum energy required. 


\begin{tabular}{|c||cccc|}
\hline Experiment & Node 1 & Node 2 & Node 3 & Node 4 \\
\hline \hline E1 & 1.0 & 1.0 & 1.0 & 1.0 \\
E2 & 0.5 & 1.5 & 1.5 & 0.5 \\
E3 & 0.4 & 0.8 & 1.2 & 1.6 \\
E4 & 0.0 & 0.0 & 1.0 & 3.0 \\
E5 & 1.6 & 1.2 & 0.8 & 0.4 \\
E6 & 3.0 & 1.0 & 0.0 & 0.0 \\
\hline
\end{tabular}

TABLE I

DESCRIPTION OF EXPERIMENTS: MAXIMUM SOURCE RATES FOR EACH NODE IN THE NETWORK

Different experiments were performed for both formulations considering variations in the node rates. The nomenclature of each experiment for the different values used for the maximum node rates $R_{i}$ is described in Table I. Normalized units are used for all parameters such as information rates and energy levels.

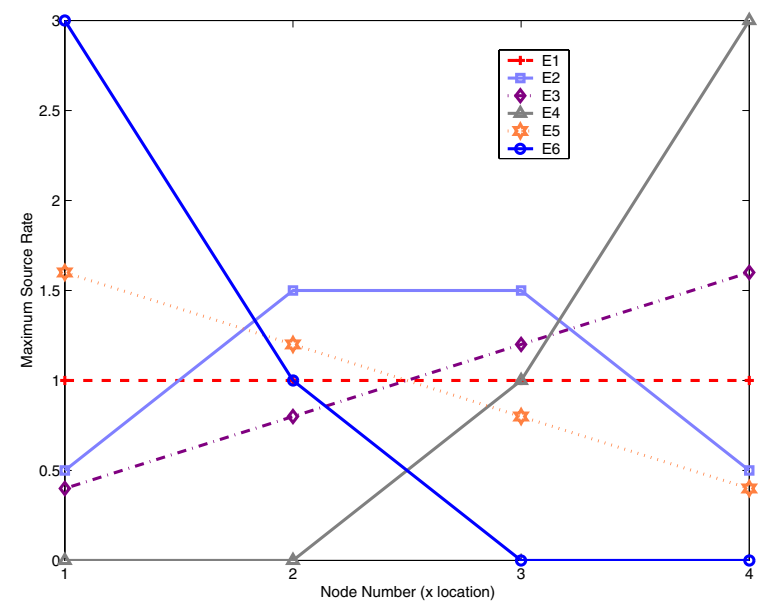

Fig. 2. Trends in the maximum source rate for each node in the network for the various experiments

These maximum node rates are also plotted in Figure 2. It can be seen from this plot that while the total source rate is kept constant in each experiment, the experiments capture different trends in the distribution of source rates (in order: constant, increasing-then-decreasing, increasing slowly, increasing sharply, decreasing slowly, and decreasing sharply).

Other problem parameters were set as follows: the noise in the communication channel $\eta=0.0001$, the reception power $C=0.00005$ (corresponds to roughly (1/5)th of the transmission power). For Problem (1) all nodes were given the same energy $E_{i}=0.0001$ units. In Problem (2) we required $f_{\text {min }}=2$ units of information.

Figure 3 shows the results of running the optimization formulation Problem (1) to maximize the information flow for each of the experiments, while varying the fairness proportion index $\alpha$. The first observation is that the total flow increases monotonically with $\alpha$. This is intuitive: as the fairness indicator $\alpha$ increases, the fairness constraint is being relaxed (recall that $\alpha=0.25$ represents the maximum fairness), allowing

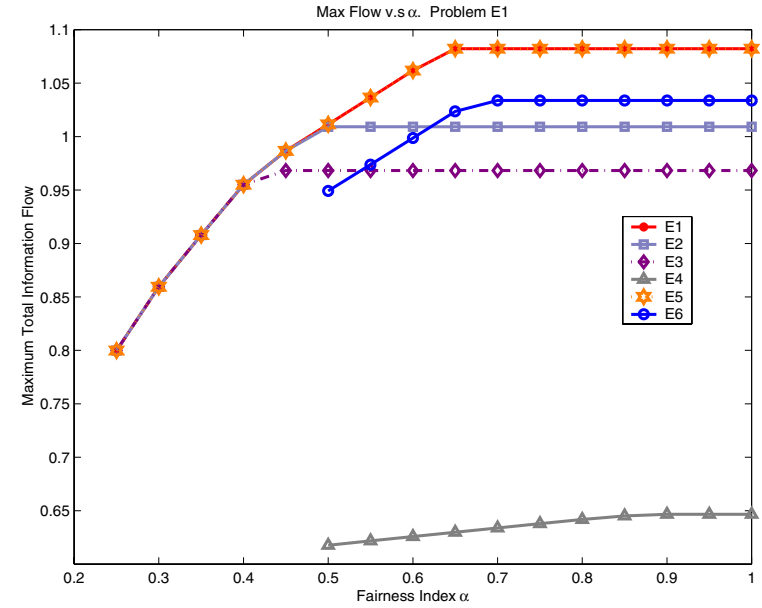

Fig. 3. Maximum total information flow to sink versus the fairness proportion index $\alpha$ for each experiment

for higher overall flow. Also note that experiments E4 and E6 begin at $\alpha=0.5$ because in these experiments only two sensor nodes have $R_{i}>0$ and can therefore send information to the sink.

Another interesting observation is that the curves for experiments E1 and E5 overlap. E5 is the experiment in which the source rates for the nodes decrease gradually with distance from the sink, while E1 has all nodes with the same source rate. The overlapping of the curves can be explained by the energy constraints. The figure suggests that the energy constraints prevent node $i$ from ever sending more than $B_{i}$ units of information, where $B_{i}=\min \left(R_{i}(E 1), R_{i}(E 5)\right)$, the minimum of the source rates for both experiments.

Similarly the curves E2 and E3 coincide with E1 when the fairness constraints are strict. However these curves do not offer overall the same flow as E1 because the information is located on average farther away than on the fixed energy experiment, therefore less of it can be drawn to the sink. E6 has less flow on the whole because greater information is located at the first node than can be drawn from it (due to a combination of both energy and fairness constraints). Finally, the flow is significantly worse for the setting E4 than all other source rate distributions. This is easy to understand - recall that in E4, most of the data is located 3 hops away from the sink, and a little 2 hops away. The limited energy budgets, combined with fairness constraints for low $\alpha$ ensure that very little of the information makes it to the sink.

Figure 4 shows the results of running the optimization formulation Problem (2) to minimize the total energy usage for each of the experiments, while varying the fairness proportion index $\alpha$. The first observation is that the minimum total energy usage decreases with respect to $\alpha$. This is intuitive: as the fairness proportion index $\alpha$ increases, the fairness constraint is being relaxed (recall that $\alpha=0.25$ represents the maximum fairness), allowing for lower energy usage. Like in the experiments with Problem (1), experiments E4 and E6 start at $\alpha=0.5$. Additionally in this formulation experiments E3 


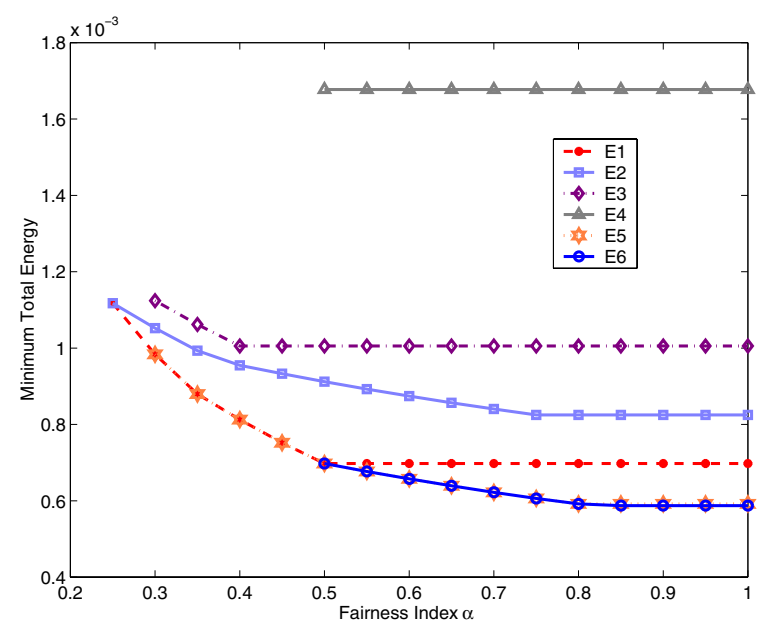

Fig. 4. Minimum total energy versus the fairness proportion index $\alpha$ for each experiment

and E5 begin with $\alpha=0.3$ because tighter fairness constraint does not allow these two cases to output 2 units of information to the sink.

We see that the curves tend to flatten out once the $\alpha$ exceeds a critical threshold - this represents the point at which the fairness constraint is no longer tight and the information constraint starts to dominate. For each strategy the beginning of this region corresponds to the energy-optimal way of extracting $f_{\text {min }}$ amount of information from the nodes. After this point the nodes have no flexibility in decreasing their energy usage because they must send the required amount of information. It can be seen that the experimental rate settings E5 and E6 lead to similar profiles for energy usage. This is because in both cases (more so in E6) the 2 units of information that need to be sent are located next to the sink. Once again E4 has the poorest performance because the information is located farthest away on average and requires greater total energy usage in order to be extracted. The curve for E4 is flat because there is no flexibility in sending information: in all cases, regardless of the fairness constraint, the optimal solution is for node 3 to send all its information ( 1 unit) and then node 4 to send an additional unit of information the sink.

Finally, we observed that Formulation (2) provides solutions that are more efficient in that these solutions can output more information using less energy. This is because the energy is distributed among the nodes in a more efficient manner in Formulation (2).

\section{Conclusions}

We are attempting to develop a comprehensive approach to understand the fundamental performance of information routing in energy-limited wireless sensor networks through formulations involving constrained non-linear optimization. We presented two such flow-based formulations in this paper - one involving maximum information extraction, and another involving minimum total energy usage.
We presented some results for a few different small-scale WSN experiments to study the solutions obtained for these problems as we vary the fairness constraints. We found that higher fairness constraints can result in significant decrease in information extraction and higher energy usage. Another observation about the results is that the flow and energy curves show qualitatively abrupt changes as the fairness constraints are varied. This has to do with the other constraints in each model (constraints on energy in Problem (1) and information extraction in Problem (2)) starting to dominate.

Although we chose a simple 5 node scenario for our experiments, we should emphasize that these formulations are reasonably tractable. Current off-the-shelf optimization software can handle problems networks involving 100 node problems in a few hours (in other experiments not presented in this paper we have tested the formulations on topologies involving a grid of 81 sensors). If required, we believe that specific algorithms can be developed to solve these formulations even faster.

Finally, we note that this is very much a work in progress. We are currently trying to make the models richer and more useful for analyzing different kinds of wireless sensor networks. One significant extension would be to incorporate innetwork aggregation to capture the data-centric nature of these systems. Other extensions we are looking into include indepth analysis of the impact of other parameters such as sensor deployment/placement, energy and information extraction constraints. In the longer term, we also hope to enhance the optimization based formulations with closed-form analytical expressions.

\section{ACKNOWLEDGMENT}

The authors would like to thank the USC Zumberge Research and Innovation Fund for the 2003-4 Interdisciplinary Research Grant that facilitated this work.

\section{REFERENCES}

[1] I. Akyildiz, , W. Su, Y. Sankarasubramaniam, and E. Cayirci, "A Survey on Sensor Networks," IEEE Communications Magazine, Vol. 40, No. 8 , pp. 102-114, August 2002.

[2] S. Toumpis and A.J. Goldsmith, "Capacity regions for wireless ad hoc networks," Submitted to the IEEE Transactions on Wireless Communications, Sept. 2001

[3] S. Toumpis and A.J. Goldsmith, "Capacity regions for wireless ad hoc networks," International Symposium on Communication Theory and Applications, 2001

[4] M. Johansson, L. Xiao and S. Boyd, "Simultaneous routing and resource allocation in CDMA wireless data networks," IEEE International Conference on Communications, Anchorage, Alaska, May 2003.

[5] M. Bhardwaj and A.P. Chandrakasan, "Bounding the Lifetime of Sensor Networks Via Optimal Role Assignments", Proceedings of INFOCOM 2002, pp. 1587-1596, New York, June 2002.

[6] K. Kalpakis, K. Dasgupta, and P. Namjoshi, "Maximum Lifetime Data Gathering and Aggregation in Wireless Sensor Networks". In the Proceedings of the 2002 IEEE International Conference on Networking (ICN'02), Atlanta, Georgia, August 26-29, 2002.

[7] A. Woo, and D. Culler, "A Transmission Control Scheme for Media Access in Sensor Networks," Mobicom 2001.

[8] R. J. Vanderbei, "LOQO user's manual - version 3.10," Optimization Methods and Software, 12:485-514, 1999. 